01

\title{
Горячая мишень. Физико-химическая модель реактивного распыления
}

\section{() В.И. Шаповалов}

Санкт-Петербургский государственный электротехнический университет „ЛЭТИ“, 197376 Санкт-Петербург, Россия

e-mail: vishapovalov@mail.ru

Поступило в Редакцию 7 июня 2018 г.

В окончательной редакции 28 января 2019 г.

Принято к публикации 7 февраля 2019 г.

Получила развитие неизотермическая физико-химическая модель реактивного распыления. Новый вариант модели описывает распыление горячей металлической мишени в смеси аргона и одного реактивного газа. Синтез пленки на всех поверхностях вакуумной камеры задан в форме поверхностной химической реакции. Для математического описания реакции использован основной постулат химической кинетики с учетом уравнения изотермы Ленгмюра и уравнения Аррениуса в неизотермических условиях. Зависимость температуры мишени от плотности тока разряда определена по результатам измерения спектров разряда в ближнем ИК-диапазоне. Для аналитического описания модели предложена система из восьми алгебраических уравнений. $\mathrm{C}$ ее помощью выполнен анализ влияния плотности тока и расхода реактивного газа на процесс реактивного распыления горячей титановой мишени в смеси аргон + азот при плотности тока $25-600 \mathrm{~A} / \mathrm{m}^{2}$. Установлено, что разогрев мишени смещает точки изменения режимов работы мишени в область меньших расходов азота и уменьшает ширину петли гистерезиса по сравнению с холодной мишенью. При этом влияние испарения мишени на процесс начинает проявляться при плотности тока более $400 \mathrm{~A} / \mathrm{m}^{2}$.

DOI: 10.21883/JTF.2019.07.47785.228-18

\section{Введение}

Пленки оксинитридов переходных металлов, имеющие широкую область применения, осаждают многими методами $[1,2]$. Среди них наиболее часто используют методы реактивного распыления на постоянном токе [3], в том числе с горячей мишенью [4-8] и высокой мощностью [9-11]. Для эффективного применения реактивного распыления необходимо установить его общие закономерности, выявить связи между управляемыми и зависимыми переменными. Эти исследования выполняют как экспериментально, так и с помощью моделирования.

Детально изучая эти процессы для холодной мишени, некоторые авторы разработали физические модели разного уровня сложности. Известны работы, начатые в 70-х г. прошлого столетия, в которых предложены частные физические модели [12-14]. Кроме этого большое число работ посвящено разработке общей модели, в которой формирование соединения на всех поверхностях вакуумной камеры представлено в виде хемосорбции [15-17].

В последнее время эту модель обновили, введя в нее дополнительный механизм формирования соединения в поверхностном слое мишени за счет имплантации ионов реактивного газа $[18,19]$. При этом в качестве механизма его формирования сохранена хемосорбция.

В работах $[20,21]$ нами предложена неизотермическая физико-химическая модель, основанная на поверхностной химической реакции. В дальнейшем эта модель была развита для процесса реактивного распыления горячей мишени [8]. Термин „горячая“ мишень введен в том смысле, что мишень может быть нагрета до температуры, при которой на фоне распыленного потока нельзя пренебрегать потоком испаренного вещества. Кроме этого на газовый разряд может оказывать значимое влияние термоэлектронная эмиссия мишени. Эта модель кратко описана в [8,22].

Цель настоящей работы состоит в изучении влияния независимых переменных, к которым относятся плотность тока разряда и расход реактивного газа, на процесс реактивного распыления горячей титановой мишени в среде $\mathrm{Ar}+\mathrm{N}_{2}$. Для этого первоначально дано подробное описание физико-химической модели реактивного распыления горячей металлической мишени в среде аргон + реактивный газ. Далее представлена полная система из восьми алгебраических уравнений, которая была использована для решения поставленной задачи.

\section{1. Система уравнений}

\section{1. Основные допущения}

Сохраним основные допущения, принятые в работе [21]. Далее обратим внимание только на эффекты, которые характерны для процесса реактивного распыления горячей металлической мишени М в газовой среде $\mathrm{Ar}+\mathrm{X}_{2}$.

Будем считать, что:

1) на всех поверхностях внутри вакуумной камеры (мишени, подложке и стенке) возникает соедине- 
ние $\mathrm{M}_{m} \mathrm{X}_{n}$. В дальнейшем физическим величинам, описывающим состояние этих поверхностей, будут присваиваться подстрочные индексы $t, s$ и $w$ соответственно. Этот процесс представим в виде поверхностной химической реакции, протекающей на $i$-й поверхности $(i=t, s, w)$, имеющей температуру $T_{i}$ :

$$
M+\frac{n}{2 m} X_{2} \stackrel{k\left(T_{i}\right)}{\longrightarrow} \frac{1}{m} M_{m} X_{n}, \quad i=t, s, w
$$

где $m$ и $n-$ стехиометрические коэффициенты; $k\left(T_{i}\right)$ константа скорости химической реакции. Реакцию (1) из газовой фазы подпитывает поток $Q_{0}$ молекул $\mathrm{X}_{2}$, введенный в камеру. В результате на каждой поверхности происходит потребление газа, которое зададим в виде потока $Q_{i},(i=t, s, w)$;

2) на поверхности мишени конкурируют два процесса:

- формирование слоя $\mathrm{M}_{m} \mathrm{X}_{n}$ по реакции (1);

- удаление слоя $\mathrm{M}_{m} \mathrm{X}_{n}$ в виде молекул за счет распыления ускоренными ионами аргона и испарения;

3) плотность тока разряда равна

$$
j=(1+\gamma) j_{+}+j_{-},
$$

где $\gamma$ - коэффициент потенциальной ионно-электронной эмиссии; $j_{+}-$плотность ионного тока; $j_{-}-$ плотность тока термоэлектронной эмиссии (формула Ричарсона-Дэшмана):

$$
J_{-}\left(T_{t}\right)=A T_{t}^{2} \exp \left(-\frac{\Delta \varphi_{t}}{k T_{t}}\right),
$$

где $A \approx 120 \mathrm{Acm}^{-2} \mathrm{~K}^{-2} ; \varphi_{t}$ - работа выхода электронов для материала мишени; $k=1.38 \cdot 10^{-23} \mathrm{~J} / \mathrm{K}-$ постоянная Больцмана.

Получим аналитическое описание физической модели в форме системы уравнений, которая даст возможность определить зависимость парциального давления реактивного газа от двух независимых переменных $p=f\left(j, Q_{0}\right)$. При выводе будем использовать уравнение кинетики реакции (1), полученное нами ранее [21] с использованием основного постулата химической кинетики. Это уравнение задает скорость изменения доли $\theta_{t}\left(0 \leq \theta_{t} \leq 1\right)$ поверхности мишени $\left(d \theta_{t} / d t\right)_{\mathrm{ch}}$, покрытой соединением $\mathrm{M}_{m} \mathrm{X}_{n}$ в виде

$$
\left(\frac{d \theta_{t}}{d t}\right)_{\mathrm{ch}}=\frac{k\left(T_{t}\right)}{N_{\mathrm{ch}}} \theta_{0 t}^{n / 2 m}\left(1-\theta_{t}\right)
$$

с константой скорости реакции по Аррениусу:

$$
k\left(T_{t}\right)=k_{0} \exp \left(-\frac{E_{a}}{k T_{t}}\right) .
$$

Другая часть поверхности мишени $\left(1-\theta_{t}\right)$ остается чистым металлом. В формулах (4) и (5): $N_{\mathrm{ch}}-$ поверхностная концентрация центров химической реакции; $\theta_{0 t}$ - относительная часть открытой поверхности мишени $\left(1-\theta_{t}\right)$, покрытая адсорбированными молекулами газа $\mathrm{X}_{2}\left(0 \leq \theta_{0 t} \leq 1\right) ; k_{0}-$ постоянная; $E_{a}-$ энергия активации химической реакции. Физическую адсорбцию молекул $\mathrm{X}_{2}$ из газа с давлением $p$ задает изотерма Ленгмюра для неизотемических условий (см. [21], формула (8)).

\section{2. Уравнение стационарного состояния поверхности мишени}

Кинетику роста пленки на поверхности мишени (рис. 1) определяют два конкурирующих процесса:

1) формирование соединения $\mathrm{M}_{m} \mathrm{X}_{n}$ по реакции (1) (поток с плотностью $J_{\mathrm{ch}}$ ). Реакция протекает на открытой части поверхности;

2) удаление $\mathrm{M}_{m} \mathrm{X}_{n}$ с части поверхности $\theta_{t}$ в виде потока с плотностью

$$
J_{C t}(j)=J_{\mathrm{Csp}}(j)+J_{\mathrm{Cev}}\left[T_{t}(j)\right] .
$$

Выражение (6) отражает удаление $\mathrm{M}_{m} \mathrm{X}_{n}$ за счет распыления $J_{\mathrm{Csp}}$ и испарения $J_{\mathrm{Cev}}$, которые по-разному зависят от плотности тока $j$.

Первый процесс идет со скоростью $\left(d \theta_{t} / d t\right)_{\mathrm{ch}}$, которую задает основной постулат химической кинетики (4) с учетом (5). Скорость процесса распыления обозначим через $\left(d \theta_{t} / d t\right)_{\mathrm{sp}}$, а испарения - $\left(d \theta_{t} / d t\right)_{\mathrm{ev}}$. Тогда кинетическое уравнение для поверхности мишени приобретает вид

$$
\frac{d \theta_{t}}{d t}=\left(\frac{d \theta_{t}}{d t}\right)_{\mathrm{ch}}+\left(\frac{d \theta_{t}}{d t}\right)_{\mathrm{sp}}+\left(\frac{d \theta_{t}}{d t}\right)_{\mathrm{ev}} .
$$

Второе слагаемое в (7) равно

$$
\frac{d \theta_{t}}{d t}=-\frac{1}{N_{\mathrm{ch}}} \frac{j-j_{\mathrm{C}}^{-}\left(T_{t}\right)}{e\left(1+\gamma_{\mathrm{C}}\right)} S_{\mathrm{C}} \theta_{t}
$$

где для соединения $\mathrm{M}_{m} \mathrm{X}_{n}: \gamma_{\mathrm{C}}-$ коэффициент потенциальной ионно-электронной эмиссии; $S_{\mathrm{C}}-$ коэффициент

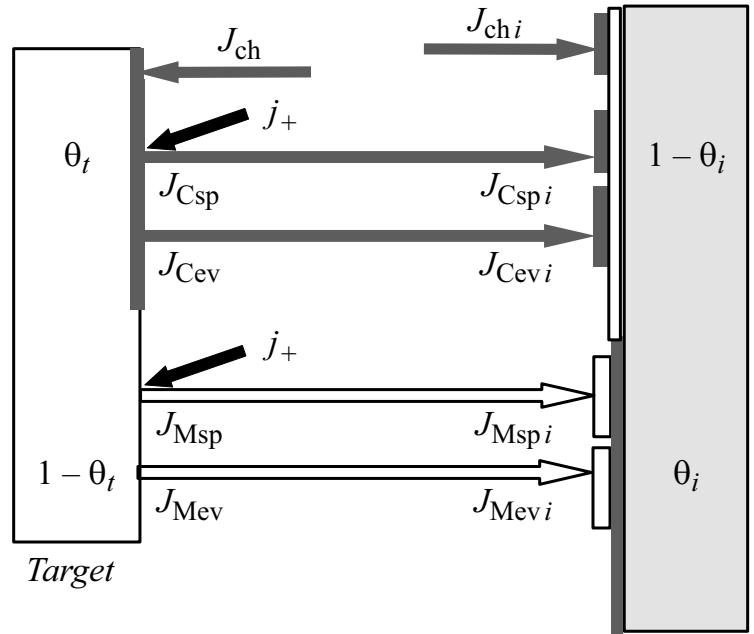

Substrate (wall)

Рис. 1. Процессы на мишени, подложке $(i=s)$ или стенке $(i=w)$. 
распыления; $j_{\mathrm{C}}^{-}-$плотность тока термоэлектронной эмиссии, которое определяет уравнение (3). Множитель в правой части (8) задает плотность ионного тока на мишени. Третье слагаемое в (7) определяется уравнением Герца-Кнудсена в форме [23]:

$$
\left(\frac{d \theta_{t}}{d t}\right)_{\mathrm{ev}}=-\frac{1}{N_{\mathrm{ch}}} \frac{10^{\left(A_{C}-\frac{B_{\mathrm{C}}}{T_{t}}\right)}}{\sqrt{2 \pi m_{C} k T_{t}}} \theta_{t},
$$

где для соединения $\mathrm{M}_{m} \mathrm{X}_{n}: A_{\mathrm{C}}$ и $B_{\mathrm{C}}-$ постоянные, определяющие давление насыщенного пара в паскалях; $m_{\mathrm{C}}$ - масса молекулы $\mathrm{M}_{m} \mathrm{X}_{n}$. Подставив в уравнение (7) выражения (4), (8) и (9), можем записать кинетическое уравнение, которое для стационарного состояния $\left(d \theta_{t} / d t=0\right)$ принимает вид

$$
k\left(T_{t}\right) \theta_{0 t}^{n / 2 m}\left(1-\theta_{t}\right)=J_{\mathrm{C}} \theta_{t},
$$

где для плотности потока $J_{\mathrm{C}}$ молекул $\mathrm{M}_{m} \mathrm{X}_{n}$ использовано общее обозначение

$$
J_{\mathrm{C}}=\left(\frac{j-j_{\mathrm{C}}^{-}\left(T_{t}\right)}{e\left(1+\gamma_{\mathrm{C}}\right)} S_{\mathrm{C}}+\frac{10^{\left(A_{\mathrm{C}}-\frac{B_{\mathrm{C}}}{T_{t}}\right)}}{\sqrt{2 \pi m_{\mathrm{C}} k T_{t}}}\right) .
$$

В дальнейшем будем оперировать с потоками атомов металла $Q_{\mathrm{M} t}$ и молекул соединения $Q_{\mathrm{C} t}$, содержащими по два компонента:

$$
\begin{gathered}
Q_{\mathrm{M} t}(j)=Q_{\mathrm{Msp}}(j)+Q_{\mathrm{Mev}}\left[T_{t}(j)\right], \\
Q_{\mathrm{Ct}}(j)=Q_{\mathrm{Csp}}(j)+Q_{\mathrm{Cev}}\left[T_{t}(j)\right] .
\end{gathered}
$$

Первые слагаемые в правых частях (11) и (12) равны распыленным потокам, вторые - испаренным. Поток (11) определен уравнением

$$
Q_{\mathrm{M} t}=J_{\mathrm{M}}\left(1-\theta_{t}\right) A_{t}=J_{\mathrm{M} t} A_{t} .
$$

В (13) для плотности потока атомов металла М использовано общее обозначение

$$
J_{\mathrm{M}}=\left(\frac{j-j_{\mathrm{M}}^{-}\left(T_{t}\right)}{e\left(1+\gamma_{\mathrm{M}}\right)} S_{\mathrm{M}}+\frac{10^{\left(A_{\mathrm{M}}-\frac{B_{\mathrm{M}}}{T_{t}}\right)}}{\sqrt{2 \pi m_{\mathrm{M}} k T_{t}}}\right),
$$

где для металла М: $\gamma_{\mathrm{M}}$ - коэффициент потенциальной ионно-электронной эмиссии; $j_{\mathrm{M}}^{-}-$плотность тока термоэлектронной эмиссии в виде $(3) ; S_{\mathrm{M}}$ - коэффициент распыления; $A_{\mathrm{M}}$ и $B_{\mathrm{M}}$ - постоянные, определяющие давление насыщенного пара в паскалях; $m_{\mathrm{M}}-$ масса атома.

Поток молекул соединения (12) равен

$$
Q_{\mathrm{C} t}=J_{\mathrm{C}} \theta_{t} A_{t}=J_{\mathrm{C} t} A_{t} \text {. }
$$

Потоки (13) и (14) переносят частицы на поверхности подложки и стенки камеры. На этих поверхностях распыление и испарение отсутствуют.

\section{3. Уравнение стационарного состояния поверхности подложки и стенки}

В стационарном состоянии часть поверхности подложки и стенки $\theta_{i}(i=s, w)$ покрыты пленкой соединения, а оставшаяся часть $\left(1-\theta_{i}\right)$ - пленкой металла. Эти состояния создают химическая реакция (1) и потоки (13) и (14), которые генерирует поверхность мишени. Формально в данном случае для описания стационарного состояния может быть использовано аналогичное уравнение для холодной мишени, подробный вывод которого дан в [21]:

$$
\begin{aligned}
& k\left(T_{i}\right) \theta_{0 i}^{n / 2 m}\left(1-\theta_{i}\right)+\frac{A_{t}}{A_{s}+A_{w}} J_{\mathrm{C}} \theta_{t}\left(1-\theta_{i}\right) \\
& =\frac{A_{t}}{A_{s}+A_{w}} J_{\mathrm{M}}\left(1-\theta_{t}\right) \theta_{i}, \quad i=s, w,
\end{aligned}
$$

где плотности потока $J_{\mathrm{M}}$ и $J_{\mathrm{C}}$ задаются с учетом особенностей распыления горячей мишени.

\section{4. Полная система уравнений}

Модель реактивного распыления как горячей, как и холодной мишени описывают восемь алгебраических уравнений [21]. К ним относятся уравнения стационарного состояния (10) и (15), три уравнения для газовых потоков на поверхности [21]

$$
Q_{i}=\frac{n}{2} k\left(T_{i}\right) \theta_{0 i}^{m / 2 n}\left(1-\theta_{i}\right) A_{i}, i=t, s, w,
$$

уравнение откачки

$$
Q_{p}=c^{0} p S_{p},
$$

где $c^{0}=2.463 \cdot 10^{25} \mathrm{~Pa}^{-1} \mathrm{~m}^{-3}-$ размерный коэффициент, переводящий поток газа $Q_{p}$, который откачивает насос, из единиц $p V$ в единицы $s^{-1} ; S_{p}$ - быстрота откачки камеры и уравнение баланса газовых потоков

$$
Q_{0}=Q_{t}+Q_{s}+Q_{w}+Q_{p}
$$

\section{2. Анализ реактивного распыления горячей титановой мишени в среде $\mathrm{Ar}+\mathbf{N}_{\mathbf{2}}$}

Система (10), (15)-(18) была решена численно для магнетрона с горячей титановой мишенью, распыляемой в среде $\mathrm{Ar}+\mathrm{N}_{2}$. Решение выполнено для функции $p=f\left(j, Q_{0}\right)$. Реакция (1) в данном случае была записана в виде

$$
\mathrm{Ti}+\frac{1}{2} \mathrm{~N}_{2} \stackrel{k\left(T_{i}\right)}{\leftrightarrow} \mathrm{TiN}, i=t, s, w .
$$

Для определения $k(T)$ в форме (5) изучен процесс реактивного магнетронного распыления холодной титановой мишени диаметром $130 \mathrm{~mm}$ в среде $\mathrm{Ar}+\mathrm{N}_{2}$. 
Таблица 1. Параметры модели реактивного распыления холодной титановой мишени в среде $\mathrm{Ar}+\mathrm{N}_{2}$

\begin{tabular}{c|c|c|c|c|c|c|c|c}
\hline Параметр & $\begin{array}{c}N_{\mathrm{ch}}, \\
10^{18} \mathrm{~m}^{-2}\end{array}$ & $a_{0}$ & $\begin{array}{c}Q_{\mathrm{ph},} \\
\mathrm{cal} / \mathrm{mol}\end{array}$ & $\tau_{a}, \mathrm{~s}$ & $S_{\mathrm{M}}$ & $S_{\mathrm{C}}$ & $\begin{array}{c}S_{p}, \\
\mathrm{~m}^{3} / \mathrm{s}\end{array}$ & $\begin{array}{c}A t, \\
\mathrm{~m}^{2}\end{array}$ \\
\hline Значение & 14.7 & $\begin{array}{c}1.0 \\
{[13]}\end{array}$ & 10000 & $10^{-13}$ & $\begin{array}{c}0.3 \\
{[24,25]}\end{array}$ & $\begin{array}{c}0.07 \\
{[25,26]}\end{array}$ & 0.025 & 0.00365 \\
\hline Параметр & $\begin{array}{c}A_{w}, \\
\mathrm{~m}^{2}\end{array}$ & $\begin{array}{c}A_{s}, \\
\mathrm{~m}^{2}\end{array}$ & $\begin{array}{c}T_{w}, \\
\mathrm{~K}\end{array}$ & $\begin{array}{c}T_{s}, \\
\mathrm{~K}\end{array}$ & $\begin{array}{c}T_{t}, \\
\mathrm{~K}\end{array}$ & $\begin{array}{c}E_{a}, \\
10^{-20} \mathrm{~J}\end{array}$ & $\begin{array}{c}k_{0}, 10^{29} \\
\mathrm{~m}^{-2} \mathrm{~s}^{-1}\end{array}$ \\
\hline Значение & 1.0 & 0.0003 & 300 & 600 & 600 & 6.5 & 1.4 &
\end{tabular}

Примечание. В таблице указаны значения параметров, задающих изотерму Ленгмюра для молекул азота (см. [21], формула (8)): $a_{0}-$ коэффициент конденсации; $Q_{\mathrm{ph}}$ - теплота физической адсорбции; $\tau_{a}-$ среднее время жизни молекулы в адсорбированном состоянии.

Таблица 2. Параметры модели реактивного распыления горячей титановой мишени в среде $\mathrm{Ar}+\mathrm{N}_{2}$

\begin{tabular}{|c|c|c|c|c|c|c|c|}
\hline Параметр & $S_{\mathrm{M}}$ & $S_{\mathrm{C}}$ & $\varphi_{\mathrm{M}}, \mathrm{eV}$ & $\varphi_{\mathrm{C}}, \mathrm{eV}$ & $\gamma_{\mathrm{M}}, \mathrm{eV}$ & $\gamma_{\mathrm{C}}$ & $A_{\mathrm{M}}$ \\
\hline Значение & $\begin{array}{c}0.30 \\
{[24,25]}\end{array}$ & $\begin{array}{c}0.07 \\
{[25,26]}\end{array}$ & $\begin{array}{l}4.5 \\
{[27]}\end{array}$ & $\begin{array}{l}4.6 \\
{[28]}\end{array}$ & $\begin{array}{r}0.053 \\
{[29]}\end{array}$ & $\begin{array}{c}0.049 \\
{[29]}\end{array}$ & $\begin{array}{c}10.4 \\
{[30]}\end{array}$ \\
\hline Параметр & $B_{\mathrm{M}}$ & $A_{\mathrm{C}}$ & $B_{\mathrm{C}}$ & $a_{0}$ & $\underset{\mathrm{cal} / \mathrm{mol}}{Q_{\mathrm{ph}}}$ & $\begin{array}{c}N_{\mathrm{ch}} \\
10^{18} \mathrm{~m}^{-2}\end{array}$ & $\begin{array}{l}k_{0}, 10^{29} \\
\mathrm{~m}^{-2} \mathrm{~s}^{-1}\end{array}$ \\
\hline Значение & $\begin{array}{c}23230 \\
{[30]}\end{array}$ & $\begin{array}{r}13.3 \\
{[31]}\end{array}$ & $\begin{array}{c}28840 \\
{[32]} \\
\end{array}$ & $\begin{array}{l}1.0 \\
{[15]}\end{array}$ & 10000 & 14.7 & 1.4 \\
\hline Параметр & $\begin{array}{c}E_{a}, \\
10^{-20}, \mathrm{~J}\end{array}$ & $\begin{array}{c}S_{p} \\
\mathrm{~m}^{3} / \mathrm{s}\end{array}$ & $\begin{array}{l}A_{t}, \\
\mathrm{~m}^{2}\end{array}$ & $\begin{array}{l}A_{w}, \\
\mathrm{~m}^{2}\end{array}$ & $\begin{array}{l}A_{s}, \\
\mathrm{~m}^{2}\end{array}$ & $\begin{array}{c}T_{w}, \\
\mathrm{~K}\end{array}$ & $\begin{array}{c}T_{s}, \\
\mathrm{~K}\end{array}$ \\
\hline Значение & 6.5 & 0.025 & 0.00365 & 1.0 & 0.0003 & 300 & 600 \\
\hline
\end{tabular}

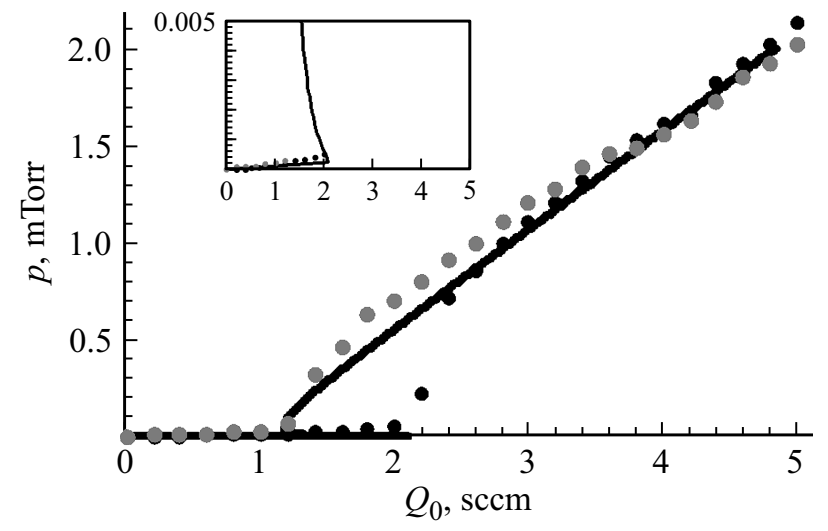

Рис. 2. Зависимость давления азота от его расхода: точки эксперимент (черные - увеличение расхода; серые - уменьшение); сплошная линия - результат численного решения уравнений (10), (15)-(18). На врезке показан график в увеличенном масштабе.

Эксперимент подробно описан в [24]. Измерены зависимости парциального давления азота от его расхода $Q_{0}$ в диапазоне 0-5 sccm при плотности тока $450 \mathrm{~A} / \mathrm{m}^{2}$. Во время измерений значения $Q_{0}$ сначала увеличивали, a затем уменьшали. Результаты измерений показаны точками на рис. 2, где наблюдается гистерезис. При решении задачи использована система уравнений (10), (15)-(18), модифицированная для частного случая хо- лодной мишени, в котором $j=(1+\gamma) j_{+}$. В этом случае изменяются плотности потоков $J_{\mathrm{C}}$ и $J_{\mathrm{M}}$ в (10) и (13), распыленные с поверхности мишени:

$$
\begin{aligned}
& J_{\mathrm{C}}=\frac{j S_{\mathrm{C}}}{e\left(1+\gamma_{\mathrm{C}}\right)}, \\
& J_{\mathrm{M}}=\frac{j S_{\mathrm{M}}}{e\left(1+\gamma_{\mathrm{M}}\right)} .
\end{aligned}
$$

При расчетах использованы параметры, указанные в табл. 1. В качестве оценочных значений коэффициентов

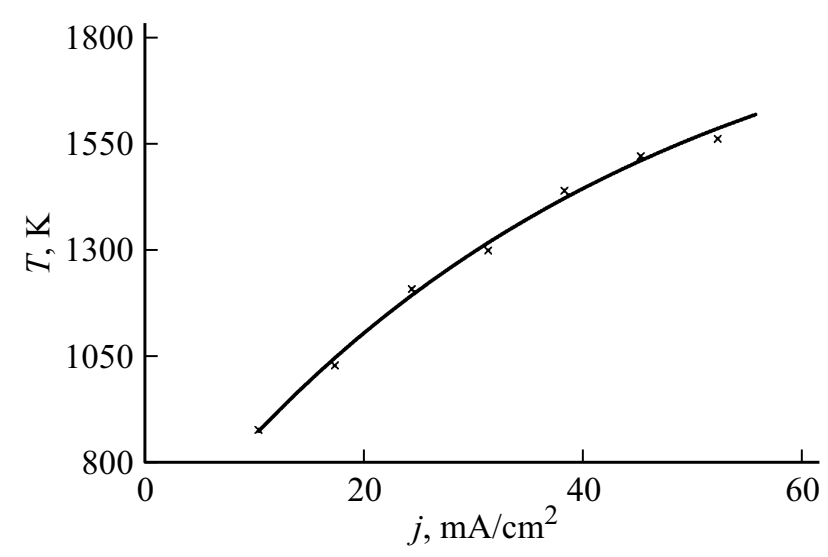

Pис. 3. Экспериментальная (точки) и модельная (сплошная линия) зависимости температуры мишени от плотности тока. 

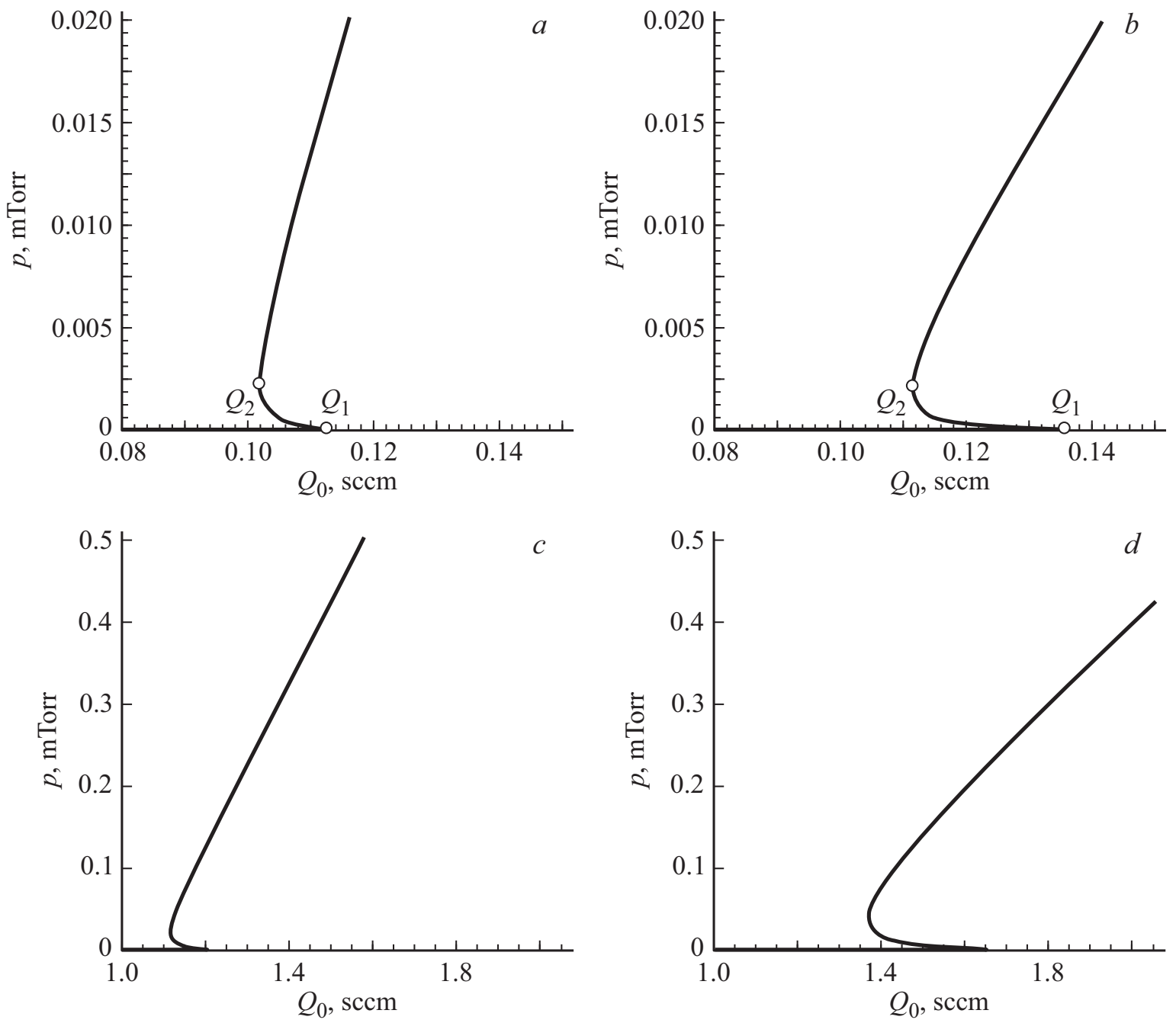

Рис. 4. Зависимости давления азота от его расхода при $j\left(\mathrm{~A} / \mathrm{m}^{2}\right): a$ и $b-50 ; c$ и $d-500$ для горячей $(a$ и $c)$ и холодной $(b$ и $d)$ мишени.

распыления пленок титана и нитрида титана при расчете использованы известные значения для объемных материалов. При вычислениях был выполнен подбор величин $k_{0}$ и $E_{a}$, которые обеспечивали наименьшее отклонение модельной зависимости от экспериментальных результатов (рис. 2) с достоверностью более 0.9. Найденные значения занесены в табл. 1 .

На втором этапе было выполнено исследование реактивного распыления горячей мишени. Табл. 2 содержит данные, которые были использованы при решении системы уравнений (10), (15)-(18). По аналогии с задачей для холодной мишени (табл. 1) в данном случае в качестве оценочных значений всех физических параметров пленок титана и нитрида титана были взяты известные значения для объемных материалов (табл. 2).

Самостоятельное значение имеет измерение температуры мишени. Чаще всего для этого применяют неконтактные инфракрасные пирометрические методы [32-34]. Но они дают значительную погрешность, поскольку пирометром интегрирует оптический спектр разряда в
ИК-области, который представляет собой комбинацию спектров испускания тлеющего разряда и теплового излучения мишени. Зависимость температуры мишени от плотности тока разряда, которая была использована при подготовке настоящей работы, определяли по методике, использованной в [35,36]. Методика основана на измерении оптических спектров разряда. При работе холодной мишени эти спектры являются линейчатыми и формируются за счет испускания фотонов возбужденными частицами разряда. Спектр разряда с горячей мишенью содержит два компонента: спектр испускания разряда и спектр излучения нагретой мишени. Методика вычисления температуры мишени построена на выделении из экспериментального спектра второго компонента. Далее производится построение теоретического спектра излучения абсолютно черного тела в пределах спектрального диапазона работы прибора с минимальной температурой. Результаты выполнения этой вычислительной процедуры показаны точками на рис. 3. 

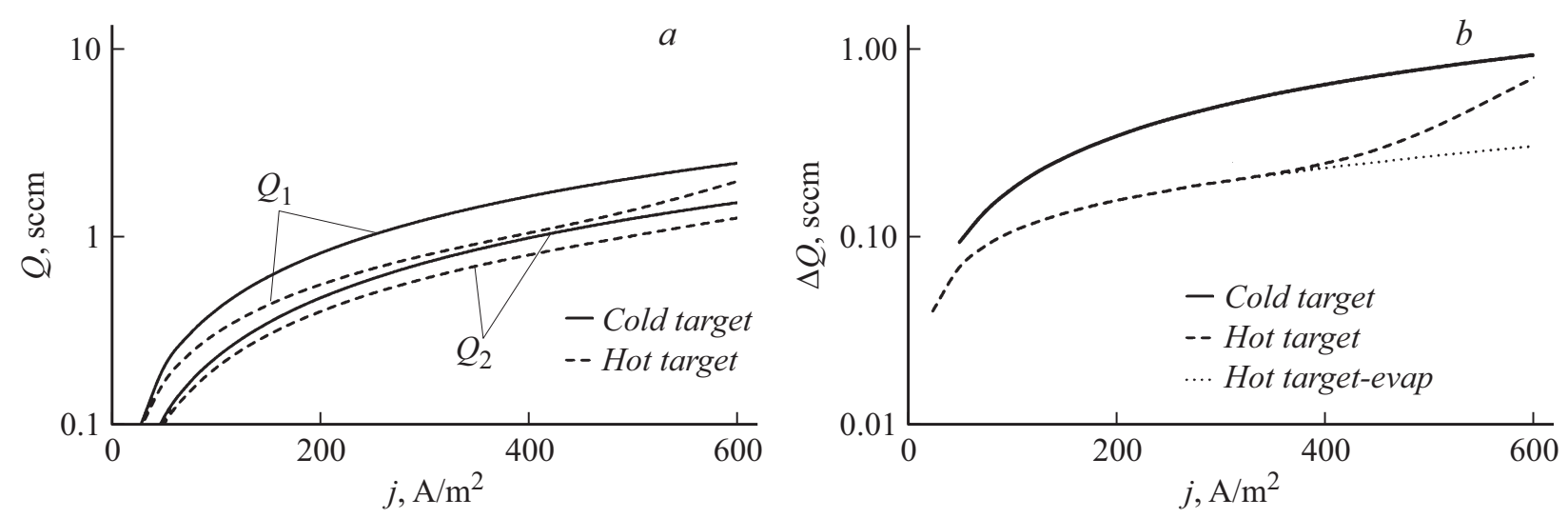

Рис. 5. Зависимости от плотности тока: $a$ - значений расхода азота, при которых происходят изменения режимов работы холодной и горячей мишени: $Q_{1}-$ из металлического режима в нитридный; $Q_{2}$ - из нитридного режима в металлический; $b$ - ширины гистерезиса.

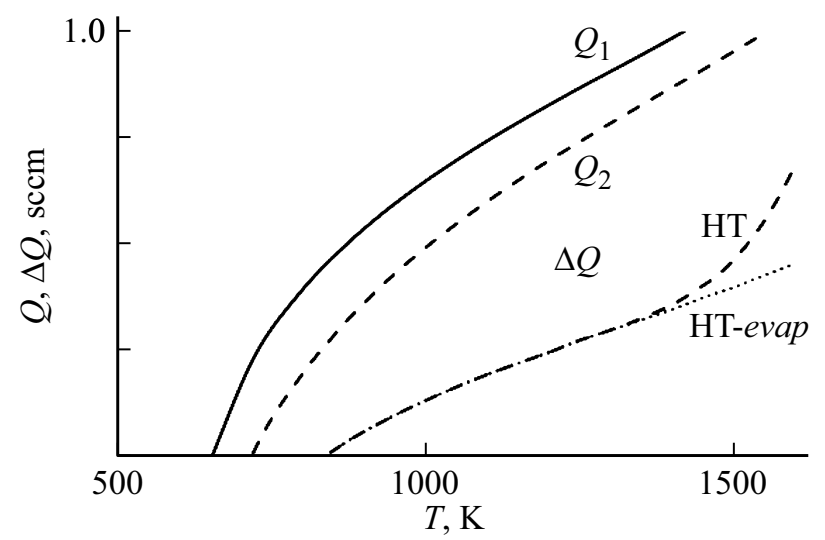

Рис. 6. Зависимости от температуры мишени ширины гистерезиса $\Delta Q$ и значений расхода азота, при которых происходят изменения режимов работы горячей мишени: $Q_{1}$ - из металлического режима в нитридный; $Q_{2}-$ из нитридного режима в металлический.

Сплошная линия на рис. 3 является результатом аппроксимации. Ее описывает выражение

$$
T_{t} \approx 2000-1440 e^{-0.00239 j} .
$$

Продолжим изучение особенностей процесса распыления горячей титановой мишени. На рис. 4, $a$, с для примера приведены зависимости $p=f\left(Q_{0}\right)$, полученные при двух значениях $j$. В каждой кривой есть две характерные точки $Q_{1}$ и $Q_{2}$. Первая соответствует переходу мишени в эксперименте из металлического режима работы в нитридный. Вторая соответствует обратному переходу. Из рис. 4, $a, c$ видно, что увеличение $j$ на порядок привело к увеличению значений $Q_{1}$ и $Q_{2}$ в восемь и десять раз соответственно. Для сравнения аналогичная задача была решена для холодной мишени (рис. $4, b, d)$.

Из рис. 4 следует, что для горячей мишени:

- эффект гистерезиса сохраняется в широком диапазоне температур. В соответствии с выражением (20) плотностям тока 50 и $500 \mathrm{~A} / \mathrm{m}^{2}$ соответствуют температуры 720 и $1560 \mathrm{~K}$;

- изменения режима работы мишени происходят при меньших значениях $Q_{1}$ и $Q_{2}$ по сравнению с холодной мишенью;

- ширина петли гистерезиса тоже имеет меньшие значения.

Результаты более детального анализа показаны на рис. 5. Рис. 5, $a$ содержит зависимости значений $Q_{1}$ и $Q_{2}$ от плотности тока для холодной и горячей мишеней, рис. $5, b$ - аналогичные зависимости ширины петли гистерезиса $\Delta Q=Q_{1}-Q_{2}$. Кроме этого на рис. 5, $b$ приведена зависимость $\Delta Q=f(j)$ (точечная кривая), полученная решением уравнений (10), (15)-(18), из которых удалены слагаемые, учитывающие испарение. Из рис. 5 видно, что отмеченные выше особенности справедливы во всем диапазоне значений $j$. Но при $j<400 \mathrm{~A} / \mathrm{m}^{2}$, что, исходя из уравнения (20), соответствует температуре мишени $1450 \mathrm{~K}$, испарение не влияет на процесс распыления. Это влияние наблюдается при $j>400 \mathrm{~A} / \mathrm{m}^{2}$. Зависимости $Q=f(j)$ и $\Delta Q=f(j)$ с рис. 5 отображены на рис. 6 , где в качестве независимой переменной принята абсолютная температура. Такая форма отражения наглядно демонстрирует возможный диапазон температур нагрева мишени и его влияние на процесс распыления.

Влияние испарения в изучаемом процессе не привело к исчезновению гистерезисного эффекта, так как это было обнаружено при анализе распыления горячей танталовой мишени в среде кислорода [11]. Это следует отнести к особенности нитрида титана, имеющего очень низкое давление насыщенного пара. На рис. 7 в широком диапазоне приведены зависимости плотности распыленного $J_{\mathrm{sp}}$ и испаренного $J_{\mathrm{ev}}$ потоков $\mathrm{TiN}$ от $j$, которые вычислены по слагаемым правой части уравнения (10) с учетом (20). Кроме оси $j$ на рис. 7 нанесена ось $T$, которая физически относится только к испаренному потоку. Утрату гистерезиса следует связать с плотностью тока разряда и температурой, при которых возникает 


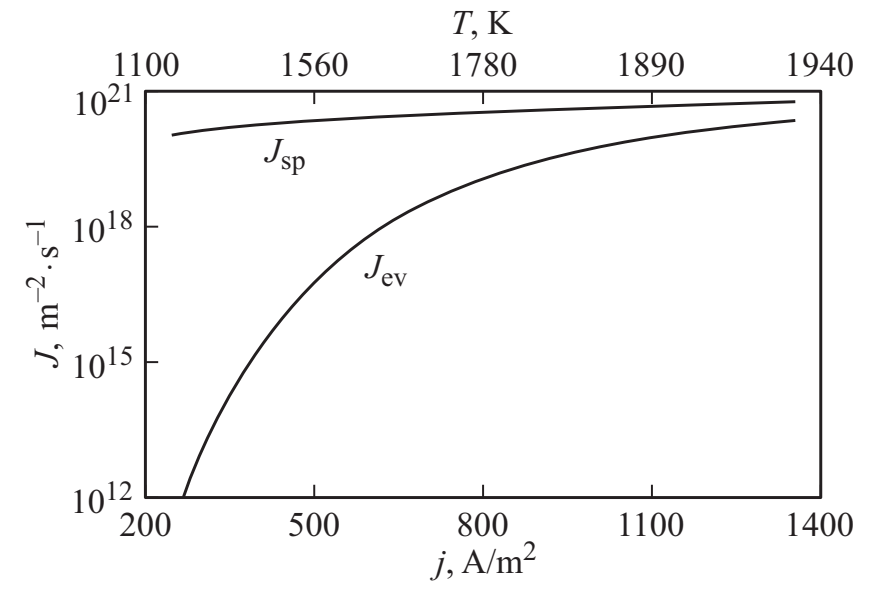

Рис. 7. Плотности распыленного $J_{\mathrm{sp}}$ и испаренного $J_{\mathrm{ev}}$ потоков с поверхности мишени.

равенство $J_{\mathrm{sp}}=J_{\mathrm{ev}}$. Как видно из рис. 7, для TiN это условие невыполнимо.

Дальнейшие вычисления позволили установить, что термоэлектронная эмиссия незначительно влияет на процесс.

\section{Выводы}

Физико-химическую модель реактивного распыления горячей металлической мишени описывает система из восьми алгебраических уравнений, задающих:

- стационарное состояние поверхностей мишени, подложки и стенки вакуумной камеры;

- газовые потоки на эти поверхности;

- откачку;

- баланс газовых потоков.

В уравнениях учтены процессы, происходящие на поверхности мишени в виде испарения и термоэлектронной эмиссии.

Численное решение этой системы дает возможность получить зависимость парциального давления реактивного газа от его объемного расхода, плотности тока разряда и выполнить детальный анализ процесса распыления. Анализ процесса распыления горячей титановой мишени в среде $\mathrm{Ar}+\mathrm{N}_{2}$, выполненный с помощью разработанной модели, позволил установить, что:

- эффект гистерезиса сохраняется во всем выделенном для исследования диапазоне значений $j$;

- изменения режимов работы мишени происходят при меньших значениях $Q_{1}$ и $Q_{2}$, чем для холодной мишени;

- горячая мишень имеет меньшую ширину петли гистерезиса, чем холодная;

- в области плотности тока менее $400 \mathrm{~A} / \mathrm{m}^{2}$ испарением можно пренебречь;

- термоэлектронной эмиссией титановой мишени можно пренебречь.

\section{Финансирование работы}

Работа выполнена при поддержке РНФ (грант № 19-19-00277).

\section{Список литературы}

[1] Shimizu T., Villamayor M., Lundin D., Helmersson U. // J. Phys. D: Appl. Phys. 2016. Vol. 49. P. 065202.

[2] Shapovalov V.I. // Glass Phys. Chem. 2010. Vol. 36. P. 121157.

[3] Borges J., Barradas N.P., Alves E., Beaufort M.F., Eyidi D., Vaz F., Marques L. // J. Phys. D: Appl. Phys. 2013. Vol. 46. P. 015305.

[4] Raman P., Shchelkanov I.A., McLain J., Ruzic D.N. // J. Vac. Sci. Technol. A. 2015. Vol. 33. P 031304.

[5] Musil J., Satava V., Baroch P. // Thin Solid Films. 2010. Vol. 519. P. 775-777.

[6] Lapshin A.E., Levitskii V.S., Shapovalov V.I., Komlev A.E., Shutova E.S., Myl'nikov I.L., Komlev A.A. // Glass Phys. Chem. 2016. Vol. 42. P. 359-362.

[7] Tesař J., Martan J., Rezek J. // Surf. Coat. Technol. 2011. Vol. 206. P. 1155-1159.

[8] Shapovalov V.I., Karzin V.V., Bondarenko A.S. // Phys. Lett. A. 2017. Vol. 381. P. 472-475.

[9] Raman P., Shchelkanov I.A., McLain J., Ruzic D.N. // J. Vac. Sci. Technol. A. 2015. Vol. 33. P. 031304.

[10] Anders A. // J. Appl. Phys. 2017. Vol. 121. P. 171101.

[11] Hecimovic A., Gudmundsson J.T. // J. Appl. Phys. 2017. Vol. 121. P. 171801.

[12] Lemperiere G., Poitevin J.M. // Thin Solid Films. 1984 Vol. 111. P. 339-349.

[13] Eltoukhy H., Natarajan B.R., Green J.E., Barr T.L. // Thin Solid Films. 1980. Vol. 69. P. 229-235.

[14] Shinoki F., Itoh A. // J. Appl. Phys. 1975. Vol. 6. P. 3381-3384.

[15] Berg S., Larsson T., Blom H.O. // J. Vac. Sci. Technol. A. 1986. Vol. 4. P. 594-597.

[16] Berg S., Blom H.O., Larsson T., Nender C.J. // J. Vac. Sci. Technol. A. 1987. Vol. 5. P. 202-207.

[17] Kozák T., Vlček J. // J. Phys. D: Appl. Phys. 2016. Vol. 49. P. 055202.

[18] Berg S., Särhammar E., Nyberg T. // Thin Solid Films. 2014. Vol. 565. P. 186-192.

[19] Särhammar E., Nyberg T., Berg S. // Sur. Coat. Technol. 2015. Vol. 279. P. 39-43.

[20] Barybin A.A., Zavyalov A.V., Shapovalov V.I. // Glass Phys. Chem. 2012. Vol. 38. P. 396-401.

[21] Barybin A.A., Shapovalov V.I. // J. Appl. Phys. 2007. Vol. 101. P. 054905.

[22] Shapovalov V.I., Smirnov V.V. // J. Phys. Conf. Ser. 2017. Vol. 857. P. 012039.

[23] Maissel L.I., Glang R. Handbook of thin film technology. NY:: McGraw-Hill, 1970. 800 p.

[24] Babinova R.V., Smirnov V.V., Useenov A.S., Kravchuk K.S., Gladkikh E.V., Shapovalov V.I., Mylnikov I.L. // J. Phys. Conf. Ser. 2017. Vol. 872. P. 012035.

[25] Anders A. // J. Vac. Sci. Technol. A. 2010. Vol. 28. P. 783-790.

[26] Lee W.H., Park S.K., Kang B.J., Reucroft P.J., Lee J.G. // J. Electron. Mater. 2001. Vol. 30. P. 84-88.

[27] Gudmundsson J.T., Brenning N., Lundin D., Helmersson U. // J. Vac. Sci. Technol. A. 2012. Vol. 30. P. 030801. 
[28] Bolotov L., Fukuda K., Tada T., Matsukawa T., Masahara M. // Jpn. J. Appl. Phys. 2015. Vol. 54. P. 04DA03.

[29] Depla D., Li X.Y., Mahieu S., De Gryse R. // J. Phys. D: Appl. Phys. 2008. Vol. 41. P. 202003.

[30] Dushman S. Scintific foundations of vacuum technique. New York-London: John Wiley and Sons Inc, 1962. 808 p.

[31] Hoch M., Dingledy D.P., Johnson H.J. // Am. Chem. Soc. 1955. Vol. 77. P. 304-306.

[32] Tesař J., Martan J., Rezek J. // Surf. Coat. Technol. 2011. Vol. 206. P. 1155-1159.

[33] Mercs D., Perry F., Billard A. // Surf. Coat. Technol. 2006. Vol. 201. P. 2276-2281.

[34] Cormier P.-A., Stahl M., Thomann A.-L. // J. Phys. D: Appl. Phys. 2010. Vol. 43. P. 465201.

[35] Shapovalov V.I., Minzhulina E.A. // Vacuum. 2019. Vol. 161. P. 324-327.

[36] Kozin A.A., Shapovalov V.I. // Sur. Coat. Technol. 2019. Vol. 359. P. 451-458. 